\title{
Radiographs Detect Dorsal Scaphoid Translation in Scapholunate Dissociation
}

\author{
Kevin Chan, MD, MSc, FRCSC ${ }^{1}$ Emil S. Vutescu, MD ${ }^{2}$ Scott W. Wolfe, MD ${ }^{2}$ Steve K. Lee, MD \\ 1 Spectrum Health, Michigan State University, Grand Rapids, Michigan \\ 2 Hospital for Special Surgery, New York, New York \\ Address for correspondence Kevin Chan, MD, MSc, FRCSC, Spectrum \\ Health, Michigan State University, 4100 Lake Dr SE, Grand Rapids, MI, \\ 49546 (e-mail: kevin.chan@spectrumhealth.org).
}

J Wrist Surg 2019;8:186-191.

\begin{abstract}
Keywords

- dorsal scaphoid translation

- scapholunate dissociation

- scapholunate interosseous ligament
\end{abstract}

Background Dorsal translation of the proximal scaphoid pole onto the rim of the distal radius is a late finding associated with chronic scapholunate instability. Dorsal scaphoid translation (DST) has been identified by magnetic resonance imaging in patients with scapholunate dissociation (SLD).

Purpose The authors proposed to determine whether DST can be reliably detected on radiographs using two different measurement techniques.

Patients and Methods Lateral radiographs of 20 patients with operatively confirmed SLD were compared with 20 uninjured patients in blinded assessment. DST was assessed using the concentric circle and dorsal tangential line methods. Reliability was calculated using intraclass correlation (ICC) values.

Results Using both techniques, the scaphoid demonstrated increased dorsal translation in patients with SLD. Inter-rater reliabilities for the concentric circles and dorsal tangential line method on radiographs had ICCs $>0.80$. Similarly, intra-rater reliabilities had ICCs $>0.90$.

Conclusions Both the concentric circles and dorsal tangential line techniques had excellent reliabilities, but the dorsal tangential line method is clinically more practical. Type of Study/Level of Evidence This is a Level III, diagnostic study.
Scapholunate interosseous ligament (SLIL) tears are the most common intrinsic wrist ligament injuries and are thought to be caused by falls or collisions on a hyperextended and pronated wrist. ${ }^{1}$ SLIL tears may lead to increased and asynchronous motion between the scaphoid and lunate known as scapholunate dissociation (SLD). Untreated, a predictable carpal instability pattern ensues, culminating in scaphoid rotary subluxation and abnormal lunate extension, or dorsal intercalated segment instability (DISI). ${ }^{2}$ SLD and DISI can initiate a slow and insidious progression to degenerative arthritis of the wrist, also known as scapholunate advanced collapse (SLAC). ${ }^{3}$

Over time, additional secondary ligamentous restraints attenuate under load, ${ }^{4}$ resulting in additional postural and kinematic deformities. ${ }^{5}$ Using three-dimensional computed tomographic models, Omori et $\mathrm{al}^{6}$ demonstrated that the proximal scaphoid pole translated dorsally and radially in three patients with chronic SLD. Increased compressive and

received

August 27, 2018

accepted after revision

December 3, 2018

published online

January 18, 2019 shear stresses from the abnormal posture and kinematics of the scaphoid cause cartilage wear on its distal and proximal poles, and progressive SLAC arthritis., ${ }^{3,8}$ Thus, reliably identifying dorsal scaphoid translation (DST) before the loss of its articular cartilage would have clinically important implications for treatment.

Recently, Meister et $\mathrm{al}^{9}$ demonstrated that magnetic resonance imaging (MRI) scans detected static DST in 13 of 18 (72\%) patients with complete SLIL tears. Using as little as $1 \%$ dorsal translation, the authors reported a sensitivity of $72 \%$ and a specificity of $100 \%$ for SLIL injury. ${ }^{9}$ While the authors contended that MRI was more accurate than radiographic analysis of dorsal translation, they did not report a technique to measure this parameter radiographically, nor were we able to identify a technique in the literature. Radiographs are universally utilized and are also obtained postoperatively to assess reduction and healing over months and

Copyright (C) 2019 by Thieme Medical Publishers, Inc., 333 Seventh Avenue, New York, NY 10001, USA. Tel: +1(212) 584-4662.
DOI https://doi.org/ 10.1055/s-0038-1677536. ISSN 2163-3916. 
years. Were it sufficiently sensitive, a technique to measure DST using radiographs would enable a convenient and more cost-effective means to identify this parameter of SLD both pre- and postoperatively.

The purpose of our study was to determine whether DST can be reliably identified and measured using radiographs in patients with operatively confirmed complete SLIL rupture and no degenerative changes. We hypothesized that radiographic DST would correlate with the presence of a complete SL ligament rupture, and describe two reliable techniques of DST measurement.

\section{Patients and Methods}

Following Institutional Review Board approval, a registry of "SLIL injuries" from three fellowship-trained hand surgeons (MGC, SWW, SKL) was retrospectively reviewed to identify eligible patients for study inclusion. Patients were included if they had adequate preoperative radiographic imaging of their injured wrists, and complete SLIL tears (all portions) confirmed operatively. Minimum radiographic examination included posteroanterior (PA) and true lateral wrist radiographs with the forearm in neutral rotation. True lateral radiographs were confirmed using the scaphopisocapitate alignment, in which the palmar cortex of the pisiform must lie within the central third of the interval between the palmar cortices of the scaphoid and capitate. ${ }^{10}$ Exclusion criteria included patients with partial SLIL tears, radiographic or operative evidence of SLAC arthritis, or inadequate imaging. The injured cohort was then matched with the same number of uninjured control patients who did not have SLIL tears, but had radiographs performed as part of routine clinical practice for undiagnosed wrist pain. No control patient was included who had abnormal SL gap, SL angle, or RL angle.

Study patients were deidentified to allow blinded radiological measurements by two independent reviewers (KC, ESV) on two separate occasions at least 4 weeks apart. Measurements performed on wrist radiographs included the SL gap, SL angle, radioscaphoid (RS) angle, RL angle, and DST. SL gap was measured on PA wrist radiographs in the midportion of the scaphoid and lunate. ${ }^{11-13}$ All other angles were measured on true lateral wrist radiographs in 0 degrees of wrist extension by tracing the longitudinal axes of the appropriate carpal bones. ${ }^{14,15}$ The scaphoid axis was represented by a tangential line connecting the palmar convexities of the proximal and distal poles. The lunate axis was perpendicular to a line between the two distal points of the lunate. The capitate axis was determined by connecting the midpoints of the proximal and distal articular surfaces.

DST was measured in two different ways on radiographs. The first method, which we have named the concentric circles method, was performed by outlining the contour of the proximal scaphoid pole and the scaphoid facet of the distal radius using an electronic circle template on the picture archiving and communication system viewer (-Fig. 1). DST was determined by measuring the distance between the centers of these two circles in a direction normal to the long axis of the radius.
The second method, which was named the dorsal tangential line (DTL) method, involved drawing a line parallel to the longitudinal axis of the radius that passes through the dorsal articular rim of the scaphoid facet of the distal radius (-Fig. 2). DST was considered to be present (positive result) if the proximal scaphoid pole translated dorsal to this line. Additionally, the amount of scaphoid translation dorsal to the dorsal tangential line $(\mathrm{mm})$ was measured in a direction normal to the tangential line.

Inter- and intra-rater reliabilities were calculated using a two-way mixed model intraclass correlation coefficient (ICC) for continuous variables. Interpretation of ICC values was based on Landis and $\operatorname{Koch}^{16}$ (- Table 1). Radiological measurements were also compared statistically using independent samples $t$-test for continuous outcomes and chi-square test for dichotomous outcomes.

\section{Results}

There were 46 complete SLIL tears in our registry. Twenty-six had inadequate imaging, leaving 20 study patients with complete SLIL tears which were compared with 20 control patients. The SLIL group had 19 males and 1 female with a mean age of 44 years (standard deviation [SD], 9). The mean time from date of injury to operation was 7 months (SD, 6). The control group had 14 males and 6 females with a mean age of 41 years (SD, 14). Neither age $(p=0.43)$ nor gender $(p=0.04)$ was statistically significantly different between the two groups once the $p$ values were adjusted for multiple statistical analyses using the Bonferroni correction.

\section{Carpal Alignment}

Patients with complete tears of the SLIL demonstrated significantly increased SL gap, SL angle, RL angle, and RS angle from the control group (-Table 2 ). These measurements are consistent with SLD and DISI.

\section{Dorsal Scaphoid Translation}

Using both the concentric circles and the dorsal tangential line methods on radiographs, there was significantly increased dorsal translation of the scaphoid in patients with SLIL tears compared with the control group (-Table 3). Mean dorsal translation in the SLIL group was $2.9 \mathrm{~mm}$ using the concentric circles method on radiographs, which was statistically increased over the mean translation of $0.9 \mathrm{~mm}$ in the control group $(p<0.001)$. Similarly, the SLIL group demonstrated a mean scaphoid translation of $2.5 \mathrm{~mm}$ dorsal to the DTL on radiographs compared with $0.6 \mathrm{~mm}$ in the control group $(p<0.001)$. Fifteen out of 20 (75\%) patients with complete SLIL tears demonstrated translation of the proximal scaphoid pole dorsal to the DTL, while only 4 of the 20 in the normal group demonstrated dorsal translation $(p<0.05)$.

\section{Reliability}

Inter-rater reliability for the concentric circles and dorsal tangential line method on radiographs was excellent on both occasions with ICC $>0.80$ ("almost perfect agreement") ( - Table 4). Similarly, intra-rater reliability for each reviewer 


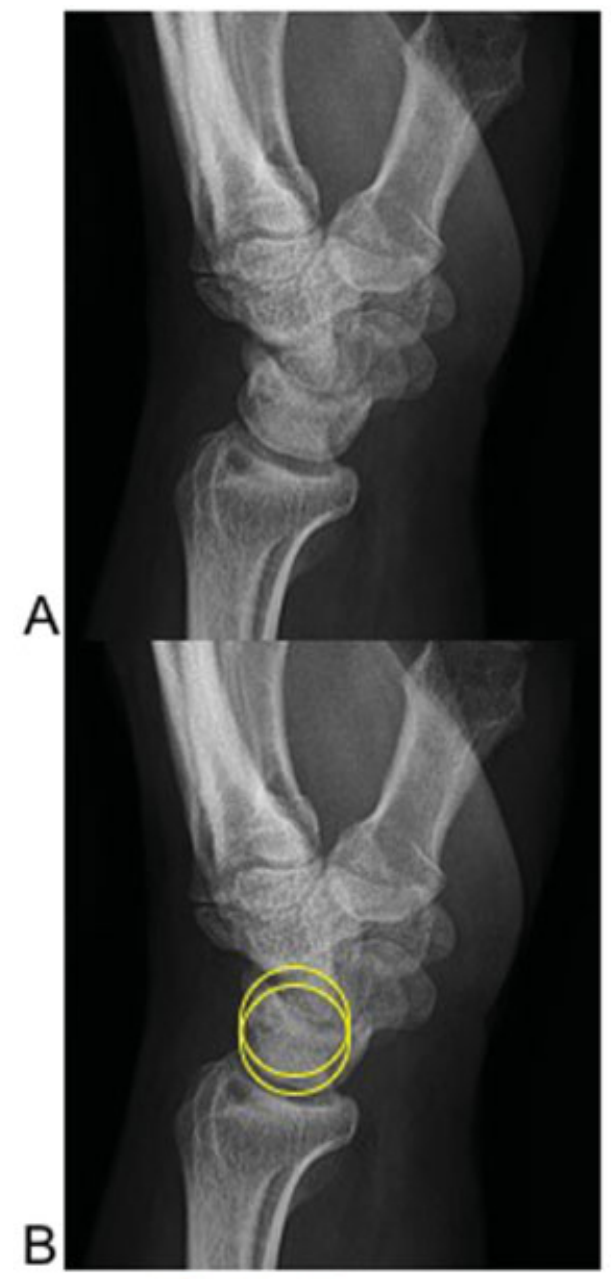

Control

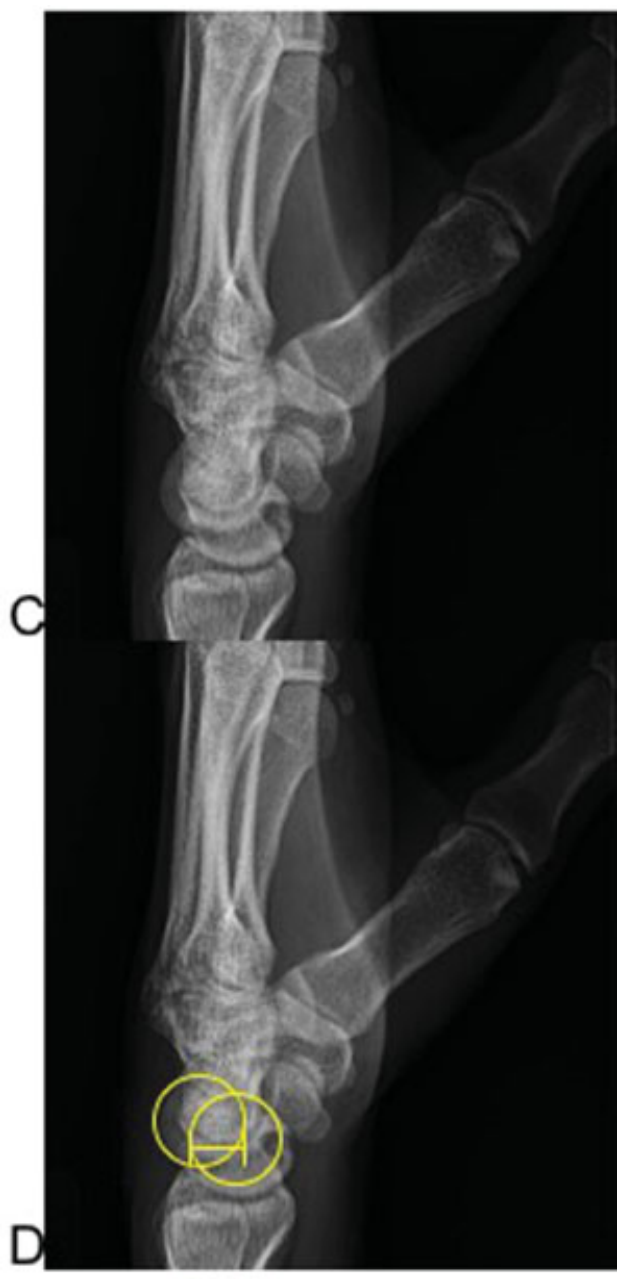

SLIL

Fig. 1 Concentric circles method-Concentric circles are drawn around the proximal scaphoid pole and the scaphoid fossa of the distal radius in patients with (A, B) normal wrist radiographs and (C, D) complete scapholunate interosseous ligament (SLIL) tears. The latter demonstrates dorsal scaphoid translation.

was also excellent with ICC $>0.90$ ("almost perfect agreement") (-Table 4).

\section{Discussion}

SLD is known to progress to carpal malalignment and ultimately, DISI. Watson et al ${ }^{17}$ described a clinical test to dynamically subluxate the dissociated scaphoid onto the dorsal rim of the radius with manual pressure on its tubercle. Ruby et $\mathrm{al}^{18}$ identified static scaphoid dorsal translation onto the dorsal rim of the radius in cadaveric wrists by dividing the SLIL, dorsal capsular ligaments, and the lunate attachment of the long radiolunate ligament (LRL). However, the concept of static DST in SLD has not been well documented clinically, and we were unable to identify techniques to measure this parameter radiographically. Radiographs are a critical imaging modality for the diagnosis and management of patients with wrist injuries. Our data demonstrate that radiographs can simply and reliably detect DST in patients with complete SLIL rupture.

Radiographic DST should be considered to complement conventional radiographic indices of SLIL injury, such as SL gap, scaphoid malrotation, and increased radiolunate angle (DISI). It should be recognized that scaphoid dorsal translation is not synonymous with SLD, rotary subluxation of the scaphoid, ${ }^{19}$ DISI, or SLAC arthritis; however, it is generally (but not always) associated with DISI and scaphoid rotary subluxation. ${ }^{19}$ Static DST is concerning, as it is diagnostic of edge-loading of the proximal scaphoid pole, ${ }^{6,9}$ and is a harbinger of future cartilage loss. ${ }^{8}$ It may precede or coexist with SLAC arthritis, depending on chronicity.

SLD is a spectrum of injury and the presence of abnormal radiographic parameters depends on the severity of SLIL rupture and the status of its secondary stabilizers. ${ }^{5} \mathrm{~A}$ recent biomechanical study demonstrated that complete division of the SLIL alone was insufficient to produce a DISI deformity; rather it required at least one secondary ligamentous stabilizer to be disrupted as well (long radiolunate, scaphotrapeziotrapezoid, or dorsal intercarpal ligaments). ${ }^{20}$ In the same study, only two of the five ligament sectioning sequences demonstrated DST, and not until the SLIL, LRL, STT (scaphotrapeziotrapezoid), and DIC (dorsal intercarpal ligament) were fully divided. This spectrum of ligamentous 


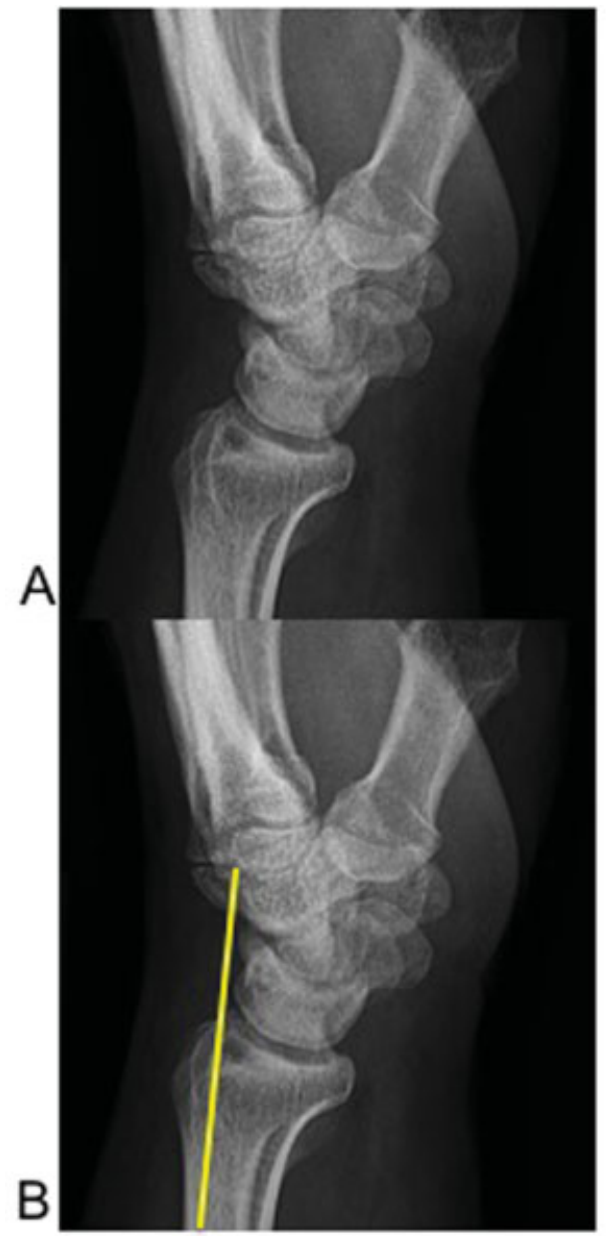

Control

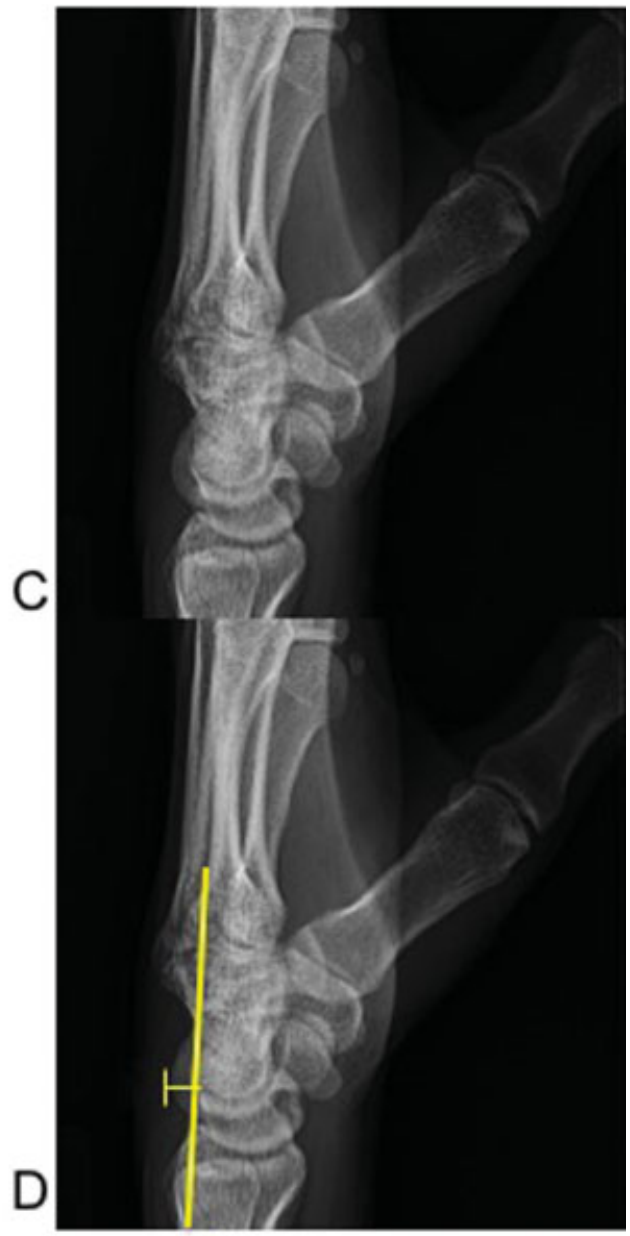

SLIL

Fig. 2 Dorsal tangential line method-The dorsal tangential line (DTL) is a longitudinal line drawn through the dorsal articular rim, parallel to the longitudinal axis of the distal radius. The DTL is drawn in (A, B) control patient and (C, D) scapholunate interosseous ligament (SLIL) tear. The proximal scaphoid pole can be seen abnormally translated dorsal to the DTL in a patient with (D) complete SLIL tear.

injury may explain why 5 out of 20 patients in our series with complete SLIL disruption did not demonstrate DST, since they might have been in earlier stages of SL instability. Thus, we feel that DST is a relatively late finding in the progression of SLD, and may be an early predictor of future SLAC arthritis.

In a prospective study of 14 patients undergoing SLIL reconstruction, the presence of DST had a high correlation

Table 1 Interpretation of intraclass correlation coefficient (ICC) values

\begin{tabular}{|l|l|}
\hline ICC & Interpretation \\
\hline$<0$ & Less than chance agreement \\
\hline $0.01-0.20$ & Slight agreement \\
\hline $0.21-0.40$ & Fair agreement \\
\hline $0.41-0.60$ & Moderate agreement \\
\hline $0.61-0.80$ & Substantial agreement \\
\hline$>0.80$ & Almost perfect agreement \\
\hline
\end{tabular}

Table 2 Comparison of carpal alignment between patients with SLIL dissociation and controls

\begin{tabular}{|l|l|l|l|}
\hline & SLIL & Controls & $p$-Value \\
\hline SL gap & $4.1 \mathrm{~mm}( \pm 1.4)$ & $2.0 \mathrm{~mm}( \pm 0.5)$ & $p<0.001$ \\
\hline SL angle & $76.7^{\circ}( \pm 11.2)$ & $50.2^{\circ}( \pm 9.5)$ & $p<0.001$ \\
\hline RL angle & $14.4^{\circ}( \pm 10.4)$ & $8.9^{\circ}( \pm 4.0)$ & $p=0.035$ \\
\hline RS angle & $63.0^{\circ}( \pm 8.7)$ & $50.0^{\circ}( \pm 11.4)$ & $p<0.001$ \\
\hline
\end{tabular}

Abbreviations: RL, radiolunate; RS, radioscaphoid; SLIL, scapholunate interosseous ligament.

Table 3 Comparison of dorsal scaphoid translation values measured on radiographs

\begin{tabular}{|l|l|l|l|}
\hline & SLIL & Controls & $p$-Value \\
\hline $\begin{array}{l}\text { Concentric } \\
\text { circles }\end{array}$ & $2.9 \mathrm{~mm}( \pm 1.7)$ & $0.9 \mathrm{~mm}( \pm 1.1)$ & $p<0.001$ \\
\hline DTL distance & $2.5 \mathrm{~mm}( \pm 1.9)$ & $0.6 \mathrm{~mm}( \pm 1.2)$ & $p<0.001$ \\
\hline DTL & $15 / 20$ dorsal & $4 / 20$ dorsal & $p<0.001$ \\
\hline
\end{tabular}

Abbreviations: DTL, dorsal tangential line; SLIL, scapholunate interosseous ligament. 
Table 4 Inter-and intrarater reliabilities of the concentric circles and dorsal tangential line measurement techniques performed on radiographs

\begin{tabular}{|l|l|l|l|l|}
\hline & Inter-rater (reading \#1) & Inter-rater (reading \#2) & Intra-rater (reader \#1) & Intra-rater (reader \#2) \\
\hline Concentric circles & 0.93 & 0.81 & 0.95 & 0.95 \\
\hline DTL distance & 0.90 & 0.92 & 0.95 & 0.99 \\
\hline DTL & 0.80 & 0.85 & 0.95 & 1.00 \\
\hline
\end{tabular}

Abbreviation: DTL, dorsal tangential line.

with postoperative pain while SL gap, SL angle, and RL angle each demonstrated no correlation. ${ }^{21}$ It is likely that static DST creates painful eccentric loading and cartilage shear between the scaphoid's proximal pole and the dorsal scaphoid facet of the radius. Static DST is the end stage of the dynamic DST that occurs in Watson's "scaphoid shift test", 17 and to the dynamic and painful scaphoid subluxation that occurs with wrist flexion following SLIL injuries ${ }^{7}$

Scaphoid dorsoradial translation was previously identified using three-dimensional bone models generated from computed tomography scans in ${ }^{6}$ six patients with SLD and DISI. The authors found that the scaphoid translation generated higher contact areas dorsoradially on the scaphoid facet of the radius.

Limitations of our study include its small sample size and retrospective design, which did not allow us to investigate clinical factors that may contribute to DST. It is probable that DST is part of the spectrum of SL instability and occurs only when secondary stabilizing ligaments are disrupted or attenuate with time. The study was not designed to measure positive and negative predictive value of DST for SLD, as examiners would be biased by concomitant radiographic findings of SLD (e.g., gap and DISI) in the affected subjects.

In conclusion, our data support our hypothesis that DST is associated with SLD and may be reliably detected radiographically using both the concentric circle and dorsal tangential line methods. The dorsal tangential line is technically easier to perform and clinically more practical than the concentric circle method, which relies on availability of digital imaging software. Either method is quickly and readily performed without advanced imaging. We believe DST is a useful radiographic parameter for SL instability that has prognostic and treatment implications, and should be recorded with measures of SL gap, SL angle, and RL angle.

\section{Note}

Investigation was performed at the Hospital for Special Surgery, 535 East 70 ${ }^{\text {th }}$ Street, New York, New York, 10021.

\section{Ethical Approval}

Ethical approval was obtained from our Institutional Review Board.

Each author certifies that he or she has no commercial associations (e.g., consultancies, stock ownership, equity interest, and patent/licensing arrangements) that might pose a conflict of interest in connection with the submitted article.

\section{Conflict of Interest}

Trimed, Inc., speaking honoraria Trimed, is the company that produces the plates and screws that Dr. Wolfe sometimes uses to fix wrist fractures. Conventus Orthopaedics, Inc., consulting fees, industry research grant, is the company that produces the Conventus Distal Radius System that Dr. Wolfe sometimes uses to fix wrist fractures. plsevier, editor, publishing royalties, is a publisher that Dr. Wolfe works with; he is editor of Green's Operative Hand Surgery and receives royalties. Extremity Medical, consulting fees, royalties, patent, is a small medical device company that Dr. Wolfe works with. They produce a novel wrist replacement that Dr. Wolfe holds a patent on and for which he receives royalties and consulting fees. Toyoba, Inc., contracted research, is a company that produces nerve conduits and contracted with Hospital for Special Surgery and Dr. Wolfe to perform animal research to test the product. Cartiva, Inc., is a company that produces synthetic cartilage and is contracting with Dr. Wolfe to direct a multicenter FDA-approved clinical trial to test the product in thumb arthritis.

Steve Lee has a patent issues for Arthrex SLAM device, for which royalty is received.

\section{Acknowledgments}

The authors would like to thank Dr. Michelle Carlson (MGC) for contributing clinical cases to our study cohort.

\section{References}

1 White NJ, Rollick NC. Injuries of the scapholunate interosseous ligament: an update. J Am Acad Orthop Surg 2015;23(11):691-703

2 Linscheid RL, Dobyns JH, Beabout JW, Bryan RS. Traumatic instability of the wrist. Diagnosis, classification, and pathomechanics. J Bone Joint Surg Am 1972;54(08):1612-1632

3 Watson HK, Weinzweig J, Zeppieri J. The natural progression of scaphoid instability. Hand Clin 1997;13(01):39-49

4 Short WH, Werner FW, Green JK, Masaoka S. Biomechanical evaluation of ligamentous stabilizers of the scaphoid and lunate. J Hand Surg Am 2002;27(06):991-1002

5 Kitay A, Wolfe SW. Scapholunate instability: current concepts in diagnosis and management. J Hand Surg Am 2012;37(10): 2175-2196

6 Omori S, Moritomo H, Omokawa S, Murase T, Sugamoto K, Yoshikawa $\mathrm{H}$. In vivo 3-dimensional analysis of dorsal intercalated segment instability deformity secondary to scapholunate dissociation: a preliminary report. J Hand Surg Am 2013;38(07):1346-1355

7 Wolfe SW. Scapholunate instability. J Am Soc Surg Hand $\cdots$ 1(01): $45-60$

8 Gondim Teixeira PA, De Verbizier J, Aptel S, et al. Posterior radioscaphoid angle as a predictor of wrist degenerative joint 
disease in patients with scapholunate ligament tears. AJR Am J Roentgenol 2016;206(01):144-150

9 Meister DW, Hearns KA, Carlson MG. Dorsal scaphoid subluxation on sagittal magnetic resonance imaging as a marker for scapholunate ligament tear. J Hand Surg Am 2017;42(09): 717-721

10 Yang Z, Mann FA, Gilula LA, Haerr C, Larsen CF. Scaphopisocapitate alignment: criterion to establish a neutral lateral view of the wrist. Radiology 1997;205(03):865-869

11 Patel RM, Kalainov DM, Chilelli BJ, Makowiec RL. Comparisons of three radiographic views in assessing for scapholunate instability. Hand (N Y) 2015;10(02):233-238

12 Loredo RA, Sorge DG, Garcia G. Radiographic evaluation of the wrist: a vanishing art. Semin Roentgenol 2005;40(03):248-289

13 Kindynis P, Resnick D, Kang HS, Haller J, Sartoris DJ. Demonstration of the scapholunate space with radiography. Radiology 1990; 175(01):278-280

14 Garcia-Elias M. Carpal instability. In: Wolfe SW, Hotchkiss RN, Pederson WC, Kozin SH, eds. Green's Operative Hand Surgery. Philadelphia, PA: Elsevier Inc.; 2011:465-522
15 Larsen CF, Mathiesen FK, Lindequist S. Measurements of carpal bone angles on lateral wrist radiographs. J Hand Surg Am 1991;16 (05):888-893

16 Landis JR, Koch GG. The measurement of observer agreement for categorical data. Biometrics 1977;33(01):159-174

17 Watson HK, Ashmead D IV, Makhlouf MV. Examination of the scaphoid. J Hand Surg Am 1988;13(05):657-660

18 Ruby LK, An KN, Linscheid RL, Cooney WP III, Chao EY. The effect of scapholunate ligament section on scapholunate motion. J Hand Surg Am 1987;12(5 Pt 1):767-771

19 Nathan R, Blatt G. Rotary subluxation of the scaphoid. Revisited. Hand Clin 2000;16(03):417-431

20 Perez AJ, Jethanandani RG, Vutescu ES, Meyers K, Lee SK, Wolfe SW. What Causes DISI? A Cadaveric Study of the Critical Ligament Stabilizers of the Intercalated Segment. 73rd Annual Meeting of the ASSH; 2018; Boston, MA

21 Lee SK, Sung K, Vutescu ES, Carlson MG, Wolfe SW. Postoperative Pain is Correlated with Scaphoid Dorsal Translation Following Scapholunate Ligament Reconstruction. Paper presented at: Proc. IWIW2017; San Francisco, CA 